INPLASY

PROTOCOL

To cite: Yu. Effectiveness and safety of different Traditional Chinese medicine therapies for Chronic atrophic gastritis: A systematic review and network meta-analysis protocol. Inplasy protocol 202090094. doi:

10.37766/inplasy2020.9.0094

Received: 26 September 2020

Published: 26 September 2020

Corresponding author:

Ting Yu

905528217@qq.com

Author Affiliation: Jiangxi University of Traditional Chinese Medicine, Nanchang, China.

Support: Grant number: 81860877, 816608.

Review Stage at time of this submission: The review has not yet started.

Conflicts of interest:

None.

\section{Effectiveness and safety of different Traditional Chinese medicine therapies for Chronic atrophic gastritis: A systematic review and network meta-analysis protocol}

Yu, Y1.

Review question / Objective: The efficacy of acupuncture, moxibustion and other traditional Chinese medicine characteristic therapies in the treatment of chronic atrophic gastritis is worthy of attention, but the efficacy and safety of acupuncture, moxibustion and western medicine in the treatment of chronic atrophic gastritis have not yet been compared, based on network meta-analysis.

Condition being studied: Considering that the methodological quality of this paper is crucial to the conclusion, we only included RCTs for TCM treatment of CAG, while TCM treatment was limited to acupuncture and moxibustion, excluding Chinese herbal medicine and massage.The research of one traditional Chinese medicine therapy and another traditional Chinese medicine or western medicine is analyzed.Comparisons between western medicines will be ruled out.

INPLASY registration number: This protocol was registered with the International Platform of Registered Systematic Review and Meta-Analysis Protocols (INPLASY) on 26 September 2020 and was last updated on 26 September 2020 (registration number INPLASY202090094).

\section{INTRODUCTION}

Review question / Objective: The efficacy of acupuncture, moxibustion and other traditional Chinese medicine characteristic therapies in the treatment of chronic atrophic gastritis is worthy of attention, but the efficacy and safety of acupuncture, moxibustion and western medicine in the treatment of chronic atrophic gastritis have 
not yet been compared, based on network meta-analysis.

Condition being studied: Considering that the methodological quality of this paper is crucial to the conclusion, we only included RCTs for TCM treatment of CAG, while TCM treatment was limited to acupuncture and moxibustion, excluding Chinese herbal medicine and massage.The research of one traditional Chinese medicine therapy and another traditional Chinese medicine or western medicine is analyzed.Comparisons between western medicines will be ruled out.

\section{METHODS}

Participant or population: Among all adults (over 18 years old) were diagnosed with CAG.

Intervention: The intervention measures are traditional Chinese medicine (limited to acupuncture, moxibustion and western medicines) for the treatment of CAG. Among them, the acupuncture includes electroacupuncture, fire acupuncture, plum flower acupuncture, etc., the moxibustion includes ginger moxibustion, suspended moxibustion, fester moxibustion, etc.

Comparator: The intervention measures are traditional Chinese medicine (limited to acupuncture, moxibustion and western medicines) for the treatment of CAG. Among them, the acupuncture includes electroacupuncture, fire acupuncture, plum flower acupuncture, etc., the moxibustion includes ginger moxibustion, suspended moxibustion, fester moxibustion, etc.

Study designs to be included: Considering that the methodological quality of this paper is crucial to the conclusion, we only included RCTs for TCM treatment of CAG, while TCM treatment was limited to acupuncture and moxibustion, excluding Chinese herbal medicine and massage.The research of one traditional Chinese medicine therapy and another traditional Chinese medicine or western medicine is analyzed.Comparisons between western medicines will be ruled out.
Eligibility criteria: RCTs for TCM treatment of CAG, while TCM treatment was limited to acupuncture and moxibustion, excluding Chinese herbal medicine and massage.

Information sources: Four English databases of PubMed, Cochrane Library, Embase, Web of Science and three Chinese databases of Chinese National Knowledge Infrastructure(CNKI), Chinese Biomedical Literature Database (CBM) and Wanfang Database (WF).

Main outcome(s): Gastroscopy and pathology.

Additional outcome(s): 1) Histological grading score.2) Helicobacter pylori clearance rate. 3) The incidence rate of adverse events.

Data management: Microsoft Excel 2016 was used to establish information data extraction table, and pre-extraction was carried out to determine the feasibility of the table. Then two team members (YT and YSF) independently extracted the following information after training: 1) Basic information: Title, author, country, year, language, etc. 2) Baseline information: Gender, age, number of persons, country, diagnostic criteria, etc. 3) Methodological information: Grouping method, allocation concealment, blind method, result bias, etc. 4) Intervention measures: Treatment measures, treatment time, frequency, etc. 5) Results: Data of primary and secondary results. After the work is completed, the results are cross-checked, if there are differences, a group discussion is conducted to determine the final result.

Quality assessment / Risk of bias analysis: Microsoft Excel 2016 was used to establish information data extraction table, and preextraction was carried out to determine the feasibility of the table. Then two team members (YT and YSF) independently extracted the following information after training: 1) Basic information: Title, author, country, year, language, etc. 2) Baseline information: Gender, age, number of persons, country, diagnostic criteria, etc. 3) Methodological information: Grouping 
method, allocation concealment, blind method, result bias, etc. 4) Intervention measures: Treatment measures, treatment time, frequency, etc. 5) Results: Data of primary and secondary results. After the work is completed, the results are crosschecked, if there are differences, a group discussion is conducted to determine the final result.

Strategy of data synthesis: 2.7.2 Network map In the network diagram, each dot represents an intervention; The larger dot area means the bigger population of the studied intervention; The line between the two dots represents that there is direct comparison to RCT studies among two interventions; The line thickness represents the numbers of direct comparison to RCT studies among two interventions. 2.7.3 Transitivity and Consistency Assessment Transitivity and consistency are the prerequisites for reticular meta-analysis. The transitivity was evaluated qualitatively from the perspective of methodology and was evaluated according to the PICO principle. Consistency was mainly to check local and overall consistency. Local consistency can be checked by loop consistency test (Higgins model). The global consistency test was verified by the corresponding inconsistency model according to different data. 2.7.4 Assessment of heterogeneity Heterogeneity tests for all included studies were performed by using Network prediction interval graph, then to study the relationship of the weighted mean difference (WMD) at a $95 \%$ confidence interval $(95 \% \mathrm{Cl})$ and estimation zone (95\%Prl) to invalid line, only when invalid line crosses perpendicularly to estimation zone but doesn't to confidence interval, then means heterogeneity exists.[25] 2.7.5 Pairwise meta-analysis If there is a direct comparison between the experimental interventions included in the data (TCM versus TCM, TCM versus placebo), the Stata14.0 will be used for Pairwise metaanalysis based on a random-effects model. 2.7.6 Network meta-analysis Two team members (YT and YSF) used statistical software - Stata (version 14.0, Stata Corporation, College Station, Texas, the
United States) for analysis. A random effects model was used for network metaanalysis to compare the variables between different interventions. By comparing Surface Under the Cumulative Ranking Curve (SUCRA), the optimum intervention measures were determined. The range of SUCRA is $0-100 \%$, the higher of the cumulative ranking curve means the better of the efficacy.

Subgroup analysis: If the analysis shows significant heterogeneity, then the root cause will be analyzed according to the PICOS principle, and the STATA 14.0 will be used for subgroup analysis.

Sensibility analysis: We will evaluate the robustness of the meta-analysis results through sensitivity analysis, and exclude Figure 1. Flow diagram of study selection process. Xiao et al. Medicine (2020) Vol:No Medicine 4 MD-D-20-06911; Total nos of Pages: 8; MD-D-20-06911 such as smallsample trials and low-quality trials to explore the impact of trial quality on efficacy estimates. In addition, we will conduct a second meta-analysis based on the results of the sensitivity analysis, summarize in tables and discuss.

Country(ies) involved: China.

Keywords: chronic atrophic gastritis, network meta-analysis, protocol.

Contributions of each author:

Author 1 - Ting Yu. 